\title{
The beta and alpha cell-specific Znt8-knockout mouse model: new mechanistic insights?
}

\author{
M. El Muayed • W. L. Lowe Jr
}

Received: 20 June 2010 /Accepted: 16 August 2010/Published online: 16 October 2010

(C) Springer-Verlag 2010

Keywords Beta cells · Insulin · Islets · Proinsulin · Slc30a8 . Znt8.ZnT8

\section{Abbreviation \\ ZnT Zinc transporter}

To the Editor: One of the most intriguing aspects of the recent article by Wijesekara at al. [1] is their experiments designed to elucidate the mechanism underlying impairment of beta cell function in the setting of decreased activity of the zinc transporter 8 (ZnT8, transcription product of the Znt8 gene, also known as Slc30a8). The authors report increased proinsulin relative to insulin in the islets and serum of beta cell-specific Znt8-knockout mice. They attribute this observation to a decrease in abundance of components of the insulin-processing machinery, namely prohormone convertase 1 and 2, as well as carboxypeptidase E.

However, the reported impaired maturation of proinsulin into insulin may alternatively suggest that ZnT8-mediated $\mathrm{Zn}$ transport into immature, proinsulin-containing vesicles facilitates the proper maturation of proinsulin to insulin. The authors consider this mechanism to be unlikely, citing a prior report by Kambe et al., which had implied that ZnT5 is the major transporter of $\mathrm{Zn}$ into the Golgi apparatus and nascent secretory vesicles [2]. Yet this was observed only in

M. El Muayed $(\bowtie) \cdot$ W. L. Lowe Jr

Division of Endocrinology, Metabolism and Molecular Medicine, Northwestern University Feinberg School of Medicine,

303 E. Chicago, Tarry15,

Chicago, IL 60611, USA

e-mail:m-muayed@northwestern.edu the setting of ZnT5 overexpression in HeLa cells, but not in beta cells natively expressing ZnT5 [2]. Furthermore, prior studies by Nicolson et al. in purified primary alpha and beta cells, as well as by us in whole primary islets, showed low levels of Znt5 mRNA (endoding ZnT5) compared with that of Znt8 [3, 4]. It is therefore uncertain whether natively produced ZnT5 really does contribute to a significant extent to $\mathrm{Zn}$ transport into nascent vesicles or the Golgi apparatus of beta cells. It is possible that ZnT8 may yet be the main transporter of $\mathrm{Zn}$ into nascent immature secretory vesicles in the absence of evidence to the contrary. Huang et al. have previously shown that the presence of $\mathrm{Zn}$ facilitates maturation of proinsulin into properly processed mature insulin, at least under certain redox conditions [5]. In contrast to the observations by Wijesekara et al. however, prior work by Nicolson et al. and Lemaire et al. showed unaltered proinsulin to insulin maturation in a global Znt8-knockout mouse model [3, 6]. These conflicting observations may be a result of variations in diet or other environmental conditions. Such a diet-dependent variation would be consistent with prior reports of variations in beta cell function between colonies of Znt8-null mice bred under different dietary conditions $[3,6]$, as discussed in a recent review [7]. Alternatively, the continued production of ZnT8 in alpha cells could, theoretically, lead to an alteration of insulin and proinsulin homeostasis in the beta cell-specific Znt8-knockout model. This would presumably occur through one or more of the many, well-known interactions between alpha and beta cells. Given the negligible level of ZnT8 in non-islet cells [8], production of ZnT8 by non-islet cells is unlikely to contribute to the phenotype.

The reported impaired proinsulin conversion is evidence of a new mechanism underlying impaired beta cell function in the setting of deficient ZnT8 activity. Several groups have 
previously attempted to address this question. It has been previously reported that neither a change in membrane potential, nor the rate of secretory vesicle exocytosis are the underlying cause of impaired beta cell function $[3,6]$. Lemaire at al. excluded a decrease in the rate of exocytosis of secretory granules or in the rate of insulin diffusion into the circulation following its release [6]. We recently reported that a decrease in ZnT8 levels in primary murine islets does not lead to decreased islet cell viability in the presence of normal or supraphysiological ambient $\mathrm{Zn}$ levels [4]. This suggests that reduced ZnT8 activity does not lead to a decrease in beta cell tolerance to elevated $\mathrm{Zn}$ levels. This provides indirect evidence that an accumulation of toxic levels of $\mathrm{Zn}$ in the cytoplasm in the setting of decreased ZnT8 function does not occur to a significant degree. Further studies examining whether increased levels of ambient $\mathrm{Zn}$ are able to compensate for partially impaired ZnT8 function would be of great value.

Acknowledgements Parts of the research upon which this commentary is based were funded by the Northwestern Memorial Foundation Center for Genetic Medicine.

Duality of interest The authors declare that there is no duality of interest associated with this manuscript.

\section{References}

1. Wijesekara N, Dai FF, Hardy AB et al (2010) Beta cell-specific Znt8 deletion in mice causes marked defects in insulin processing, crystallisation and secretion. Diabetologia 53:1656-1668

2. Kambe T, Narita H, Yamaguchi-Iwai Y et al (2002) Cloning and characterization of a novel mammalian zinc transporter, zinc transporter 5 , abundantly expressed in pancreatic beta cells. J Biol Chem 277:19049-19055

3. Nicolson TJ, Bellomo EA, Wijesekara N et al (2009) Insulin storage and glucose homeostasis in mice null for the granule zinc transporter ZnT8 and studies of the type 2 diabetes-associated variants. Diabetes 58:2070-2083

4. El Muayed M, Billings LK, Raja MR et al (2010) Acute cytokine mediated downregulation of the zinc transporter ZnT8 alters pancreatic beta cell function. J Endocrinol 206:159-169

5. Huang XF, Arvan P (1995) Intracellular transport of proinsulin in pancreatic beta-cells. Structural maturation probed by disulfide accessibility. J Biol Chem 270:20417-20423

6. Lemaire K, Ravier MA, Schraenen A et al (2009) Insulin crystallization depends on zinc transporter ZnT8 expression, but is not required for normal glucose homeostasis in mice. Proc Natl Acad Sci USA 106:14872-14877

7. Rutter GA (2009) Think zinc: new roles for zinc in the control of insulin secretion. Islets 2:49-50

8. Murgia C, Devirgiliis C, Mancini E, Donadel G, Zalewski P, Perozzi G (2009) Diabetes-linked zinc transporter ZnT8 is a homodimeric protein expressed by distinct rodent endocrine cell types in the pancreas and other glands. Nutr Metab Cardiovasc Dis $19: 431-439$ 